\title{
Explaining and predicting online tourists' behavioural intention in accepting consumer generated contents
}

\begin{abstract}
Purpose: The purpose of this study is to understand the factors influencing Iranian tourists' behavioural intention to use consumer-generated contents (CGC) websites whilst browsing the web when it comes to travel planning, based upon the Technology Acceptance Model (TAM) extension. Design/methodology/approach: The total of 211 valid responses were collected through an online questionnaire survey. Data collected from Iranian online tourists were used to test the proposed research model by using the partial least squares structural equation modeling method of analysis. Findings: The results of the study indicate that perceived source credibility is the strongest predictor for behavioural intention. Furthermore, in this study, the most significant relationship is between perceived enjoyment and perceived ease of use. However, the findings of this study show that perceived ease of use is unable to predict behavioural intention of Iranian tourists while using CGC websites. Research limitations/implications: This study provides clear evidence for the importance of additional key variables in improving the authors' understanding of the Iranian adoption behaviour concerning CGC websites for travel planning purposes. Implications of this research can aid both academicians and practitioners towards a better understanding of tourists' adoption behaviour when associated with the social media. Originality/value: This paper adopts TAM and extends it with other variables, namely, perceived enjoyment, perceived source credibility and perceived risk. It examines their influence in the behavioural intention of using CGC for travel planning in the context of Iran for the first time.
\end{abstract}

Keyword: Social media; Technology acceptance model; Consumer-generated content; Behavioural intention; Iranian online tourists 